\title{
Importancia del análisis del comportamiento higrotérmico de inmuebles históricos para la rehabilitación energética. Caso del archivo histórico general del estado de Sinaloa
}

Importance of the analysis of the hygrothermal behavior of historic buildings for energy rehabilitation. Case of the general historical archive of the state of Sinaloa

Recibido: agosto 2020

Aceptado: febrero 2021

\section{Resumen}

El presente artículo tiene como objetivo contribuir al área de conocimiento sobre el comportamiento higrotérmico de inmuebles históricos en México, así como también, exponer la importancia que tienen este tipo de análisis como una herramienta útil para llevar a cabo intervenciones de rehabilitación energética para los inmuebles en cuestión. Esto con la finalidad de mejorar la eficiencia energética cooperando con la sustentabilidad de los centros urbanos y su conservación. Partiendo de los resultados obtenidos del análisis del comportamiento higrotérmico de un caso de estudio sobre un inmueble histórico ubicado en la ciudad de Culiacán, México, se pretende demostrar cómo por medio de dicho análisis es posible el diagnóstico de temperatura y humedad relativa, contribuyendo a la toma de decisiones sobre las estrategias que podrían ser utilizadas que ayuden a disminuir el consumo de energía eléctrica, mientras se mantienen condiciones higrotérmicas confortables, y se respetan los valores patrimoniales del inmueble.

\section{Palabras Claves:}

rehabilitación energética; conservación; confort higrotermico
Catalina Borbolla Gaxiola ${ }^{1}$

\begin{abstract}
This article has as purpose contribute to the area of knowledge on the hygrothermal behavior of historical buildings in Mexico, as well as expose the importance of this type of analysis as a useful tool to carry out energy rehabilitation interventions in this kind of buildings This in order to improve energy efficiency to cooperate with the sustainability of urban centers and their conservation. Based on the results obtained from the analysis of the hygrothermal behavior of a case study on a historical buildings with heritage values located in the city of Culiacán, México, the aim is to demonstrate how, through the analysis of hygrothermal behavior, it is possible to diagnose the hygrothermal conditions, contributing to decision-making about the strategies that could be used that help reduce the consumption of electrical energy, while comfortable hygrothermal conditions are maintained, and the built heritage values are respected.
\end{abstract}

\section{Keywords:}

energy rehabilitation; conservation; hygrothermal comfort

\footnotetext{
${ }^{1}$ Nacionalidad: mexicana; adscripción: Universidad Autónoma de Sinaloa; Doctorado Ciudad, Territorio y Sustentabilidad de la Universidad de Guadalajara; correo: catalinabg91@gmail.com
} 


\section{INTRODUCCIÓN}

Actualmente en México, no está normado que las acciones para la conservación de los inmuebles históricos consideren los parámetros de confort ambiental que requieren los usuarios de acuerdo a los nuevos usos de estos inmuebles y las actividades que en ellos se realizan. Así mismo, tampoco es un requisito regulado la implementación de sistemas o estrategias para la mejora de la eficiencia energética. La ley mexicana encargada de la investigación, protección, conservación, restauración y recuperación de este tipo de inmuebles, denominada Ley Federal sobre Monumentos y Zonas Arqueológicos, Artísticos e Históricos, no establece lineamientos en sus artículos referentes a los monumentos históricos, sobre la importancia de considerar la rehabilitación energética como parte fundamental en la conservación de este tipo de inmuebles. Esto, aunado a los cambios de uso que sufre el inmueble cuando la función original se vuelve obsoleta, y la alteración de sus estructuras técnicas para satisfacer las necesidades de confort ambiental de los usuarios, ocasiona que su conservación se dificulte y se presente un conflicto entre los requerimientos de la actualidad y el respeto a los valores patrimoniales.

El valor patrimonial atribuido a los inmuebles históricos ayuda a fortalecer la historia del lugar. Además, funcionan como puentes que conectan lugares tangibles y tradiciones culturales intangibles, por lo que deben ser conservados como patrimonio de la sociedad (Liudmila y Balkiz, 2019). El patrimonio se considera un recurso fundamental en los sistemas urbanos donde las intervenciones de rehabilitación deberían incluir los elementos tangibles e intangibles, así como la calidad de vida de sus habitantes. Por lo tanto, la eficiencia energética de los edificios ubicados dentro de los centros históricos es fundamental para garantizar la comodidad y el bienestar de sus habitantes (Gregório y Seixas, 2017).

La rehabilitación energética de inmuebles históricos podría no únicamente mejorar el consumo energético y los niveles de confort higrotérmico, también podría convertirse en una herramienta que permita mejorar su conservación, puesto que cuando se combina con las políticas de preservación para edificios dotados de atributos culturales o históricos, la rehabilitación arquitectónica retiene y promueve un importante capital social: el patrimonio construido
(Muranim y Ghisi, 2016). Además, puede ser una alternativa para reducir los impactos ambientales causados por la industria de la construcción (Ibídem), no solamente aquellos ocasionados por las actividades y uso de recursos al realizar nuevas construcciones, sino también a las actividades de demolición de las construcciones existentes (Ibídem). La construcción genera aproximadamente 136 millones de toneladas de residuos al año, y aproximadamente la mitad proviene de la demolición (Yung \& Chan, 2012).

La importancia de la rehabilitación energética de inmuebles históricos puede percibirse en su contribución a la sustentabilidad en los centros urbanos, la delación de los valores patrimoniales, y en extender la vida útil del inmueble al apoyar su conservación como instrumento que permita un reúso adaptativo de los inmuebles. No obstante, De Bouw, Dekeyser, Dubois y Vanhellemont (2016) han identificado que las intervenciones de rehabilitación energética en edificaciones históricas se encuentran determinadas por cuatro problemáticas principales:

1. La conciliación entre el ahorro energético/ optimización de la comodidad y los valores del patrimonio es vista como una tarea muy difícil o inclusive imposible

2. Aún existe un desconocimiento importante sobre la aplicación de nuevas tecnologías, sobre el nivel o grado de daño o beneficio que éstas podrían llegar a generar.

3. Falta investigación sobre el comportamiento y la reacción de las nuevas tecnologías al paso del tiempo.

4. La idea más recurrente en la conservación patrimonial, es que el estado original del inmueble debe ser restaurado por materiales y métodos de construcción tradicionales en la medida que sea posible.

Siendo un obstáculo importante el desconocimiento en esta área de estudio, ya sea referente a la aplicación de nuevas tecnologías y sus beneficios o posibles daños a largo plazo, o al comportamiento ambiental del inmueble propio de sus materiales y sistemas constructivos, se ha visto la necesidad de diseñar estrategias para la evaluación de los inmuebles históricos, ya sea a nivel ambiental, de consumo energético, o midiendo su generación de $\mathrm{CO}_{2}$ Una de estas estrategias es el análisis del confort higrotérmico.

Este artículo tiene como objetivo exponer la utilidad del análisis higrotérmico en inmuebles 
históricos por medio de la monitorización, como herramienta de evaluación y diagnóstico del comportamiento ambiental del inmueble, permitiendo la generación de información para apoyar la toma de decisiones sobre las intervenciones necesarias de rehabilitación energética para que las condiciones ambientales sean las más adecuadas para los usuarios, promuevan la eficiencia energética en el inmueble y permitan la conservación de sus valores patrimoniales.

\section{ESTADO DEL ARTE}

El campo de estudio de las rehabilitaciones energéticas de inmuebles históricos abarca gran cantidad de temáticas: la eficiencia energética, el reúso adaptativo de dichos inmuebles, el estudio de las características de confort ambiental presente en los espacios interiores, el respeto a los valores patrimoniales al realizar las intervenciones, diseño de mecanismos de evaluación para la toma de decisiones, como lo sugiere la investigación de López-Zambrano, Canivell y Calama-González (2019). Claesson y Brostöm (2016), y Santos, Valenca y Sequeira (2014), sostienen que en la rehabilitación energética de edificios históricos existen dos retos principales: obtener estándares modernos de confort mientras se preservaba la identidad histórica y cultural. Mientras investigaciones como las de Epelde (2015) y Lucchi, Garegnani, Maturi y Moser (2014) exponen la importancia de la adecuación de los edificios históricos tomando en cuenta la estética original del inmueble, señalando como principal problemática que la apariencia de los contextos donde se localizan los inmuebles históricos no es compatible con la apariencia de nuevas tecnologías, o con la estética y diseño de las superficies actuales.

Una rehabilitación energética puede componerse de tres etapas principales: evaluación y diagnóstico del inmueble, toma de decisiones respecto a las estrategias a seguir de acuerdo a los lineamientos de conservación patrimonial, y la evaluación del desempeño de las posibles estrategias antes de ser aplicadas, por medio del uso de software computacionales, para después pasar a la aplicación real de las estrategias en el inmueble (Borbolla, 2017). Considerando el objetivo del presente artículo, se hará énfasis en la revisión de investigaciones donde fuera considerada la primera etapa, puesto que la evaluación y diagnóstico del inmueble es fundamental para desarrollar las etapas consecutivas.

Curtis (2016), propone un enfoque de reacondicionamiento de inmuebles históricos donde trabaja con materiales tradicionales y la estructura existente. Para evaluar el desempeño térmico de las paredes y otras áreas del inmueble empleó medidores de flujo de calor in situ, los cuales son sensores que miden el flujo de energía a través de una superficie. Evaluar cuánto calor se pierde a través de paredes externas es clave para construir simuladores de energía, y es fundamental para asegurar la calidad y la toma de decisiones en la implementación de políticas y diseño de edificios, su construcción y su renovación (Sassine, 2016).

De acuerdo a Troi (2016), en su mayoría, las decisiones de reacondicionamiento no son meramente energéticas, sino económicas, pero el confort ambiental juega un papel importante. Presenta tres casos de estudio sobre el reacondicionamiento de inmuebles históricos, emplea la monitorización para la evaluación del consumo de energía y la comodidad del usuario, así como las condiciones de la estructura del edificio. Dubois, De Bouw, Vanhellemont, Stiernon y Trachte (2018) también emplean la monitorización con redes de sensores inalámbricos conocidos como data loggers, para modelar el comportamiento higrotérmico de inmuebles históricos.

Otro ejemplo, es el trabajo de Wessberg, Leijonhufvud y Brostöm (2016), el cual consistió en evaluar y comparar tres métodos de acondicionamiento para interiores. Se requirió de una monitorización previa para analizar el clima interior del inmueble, donde se midió la temperatura y la humedad relativa (Broström, Tor and Leijonhufvud, Gustaf, 2010). Krus, Kilian y Pfundstein (2016), analizan distintos tipos de aislamiento interno para muros, con el fin de reacondicionar los edificios históricos, para lo que se necesitó obtener mediciones con sensores de temperatura, humedad y flujo de calor.

Para la evaluación y diagnóstico del comportamiento higrotérmico de inmuebles patrimoniales, también se combinan métodos. Muñoz, León y Navarro (2014), para el diseño y evaluación de un sistema de climatización que mejore las condiciones de confort térmico y la preservación del patrimonio (bienes muebles) de estos edificios, se apoyaron de la monitorización y de modelos informáticos de simulación. 
Espinoza, Quesada, Calle y Ortiz (2019), evalúan los estándares de eficiencia energética y la calidad del ambiente de viviendas patrimoniales. Combinaron el uso de encuestas y entrevistas sobre la percepción del ambiente interior y el confort higrotérmico, coincidiendo la información recopilada con datos obtenidos por medio de monitorización de variables ambientales con equipos instalados en el sitio.

Las rehabilitaciones energéticas de inmuebles históricos se fundamentan por medio de tres principios: la mejora en el consumo energético considerando los parámetros de confort ambiental, realizar las adecuaciones necesarias intentando respetar lo más posible a la estética original del inmueble, para contribuir a la conservación de los valores patrimoniales intrínsecos a dichos inmuebles. La evaluación y diagnóstico del comportamiento higrotérmico de los espacios interiores es considerada una etapa de gran importancia, y ya sea combinando métodos de análisis y recopilación de información, el uso de instrumentos de medición in situ se presenta como un método que otorga resultados confiables, al que múltiples investigadores suelen recurrir.

La relación entre eficiencia energética, las condiciones higrotérmicas y el respeto a los valores patrimoniales

La importancia del estudio de las condiciones higrotérmicas de inmuebles históricos al realizar una rehabilitación energética, puede ser señalada en relación a la eficiencia energética, y su contribución a la conservación de estos inmuebles por medio del respeto de sus valores patrimoniales. Egusquiza, Prieto, Izkara y Bejar (2018) sostienen que la forma en que los edificios históricos abordan las condiciones ambientales para proporcionar condiciones de confort a los usuarios debe considerarse parte de su valor cultural y patrimonio técnico.

Rehabilitación energética se entiende como "el conjunto de actuaciones para la adecuación del parque edificatorio existente que tienen como finalidad mejorar su comportamiento energético" (Granados, 2014, p. 04). No obstante, la rehabilitación energética de inmuebles históricos, debe tener por objetivo respetar los valores patrimoniales que tienen este tipo de inmuebles.

La eficiencia energética, es definida por el Consejo Mundial de Energía como "una reducción en la energía utilizada para un servicio de energía dado (calefacción, iluminación, etc.) o nivel de actividad" (World Energy Council, 2014: 03). La Ley para el Aprovechamiento Sustentable de la Energía menciona que la eficiencia energética son "todas las acciones que conlleven a una reducción, económicamente viable, de la cantidad de energía que se requiere para satisfacer las necesidades energéticas de los servicios y bienes que demanda la sociedad" (p. 26). Puede ser entendida como el uso de la energía para satisfacer las necesidades de confort de las personas, apoyándose de las tecnologías para disminuir la cantidad empleada.

Las necesidades de confort de las personas se presentan cuando nuestro cuerpo percibe sensaciones físicas, estas pueden ser traducidas por el cerebro como placenteras o no placenteras. Se determinan por una serie de factores, $y$, cuando existe una predominancia de sensaciones placenteras, se dice que las personas se encuentran en confort.

El confort de un ambiente dependerá de dos particularidades: los parámetros ambientales de confort y de los factores de confort del usuario (Serra y Coch, 1995). Los parámetros ambientales son aquellas características físicas y ambientales de un espacio, las cuales son clasificadas en específicas y generales. Los parámetros específicos, pueden ser térmicos, acústicos o visuales, generando el confort acústico, confort visual y confort climático, el cual se divide en calidad del aire y en confort térmico. Este se convierte en un factor de gran importancia que interviene en la comodidad de las personas, por lo tanto, el confort higrotérmico sería fundamental buscar lograr un espacio confortable (Ibídem). González Licón (2006) define al confort térmico como las condiciones de bienestar de las personas, desde el punto de vista de equilibrio con las condiciones de temperatura y humedad de un sitio determinado.

Por lo tanto, el confort higrotérmico resulta fundamental para el uso de cualquier espacio de forma eficiente y satisfactoria, siendo un aspecto que definiría el aumento o disminución del uso de energía, y la instalación de mecanismos de enfriamiento o calefacción. No obstante, estas variables podrían ser reducidas empleando estrategias de diseño pasivas y conociendo las condiciones de confort aceptables por los usuarios (Attia \& Carlucci, 2015, citado por Jara, 2015). Sin embargo, las obligaciones para el confort térmico podrían entrar en conflicto con 
las de conservación de los inmuebles históricos, y por lo tanto se necesitaría llegar a un equilibrio (Muñoz et al., 2014). Es justo en este aspecto donde se sitúa gran parte del debate referente a la rehabilitación energética de inmuebles históricos.

Josep Ballart (2002) menciona que al patrimonio histórico tiene tres valores distintos: El valor de uso, el valor formal, y el valor simbólico significativo. Aloïs Riegl (1987) sostiene que los inmuebles patrimoniales presentan cinco valores: el de la antigüedad, el histórico, el rememorativo intencionado, el instrumental y el artístico. El respeto de estos, se logra por medio de la conservación, entendida como "todas aquellas medidas o acciones que tengan como objetivo la salvaguarda del patrimonio cultural tangible, asegurando su accesibilidad a generaciones presentes y futuras" (ICOM, 2008: 01).

El reto reside en buscar aquellas medidas o acciones por medio de las cuales sea posible la delación de los valores patrimoniales, sin embargo, se requiere adaptar los inmuebles históricos para los usos actuales, donde la presencia de un confort higrotérmico es fundamental. Al realizar estudios sobre el comportamiento higrotérmico de inmuebles históricos se estaría contribuyendo a la generación de evidencia e información útil para la toma de decisiones sobre las acciones a realizar.

\section{METODOLOGÍA}

Siendo el objetivo del presente artículo mostrar la importancia de realizar análisis higrotérmicos en inmuebles históricos como una herramienta clave en la toma de decisiones para su conservación, se muestra la metodología empleada en un caso de estudio ubicado en Culiacán, México, zona del país con clima tipo BS1(h')w, el cual corresponde a climas cálidos y muy cálidos, con temperatura media anual mayor a $18^{\circ} \mathrm{C}$, y por su humedad se clasifica como clima semiárido, con un régimen pluvial de lluvias en verano.

Fue analizado el edificio del Archivo Histórico General del Estado de Sinaloa, cuya construcción data del periodo colonial. Construido originalmente de una sola planta con muros de adobe, actualmente se dispone en dos plantas compuesto por sistemas constructivos variados, tanto antiguos como actuales, con una tipología de patio central (Figura 1). La envolvente de la edificación está compuesta de mampostería de ladrillo de dimensiones $07 \times 24 \times 40 \mathrm{~cm}$., con aplanado de mortero de cal arena. Gran parte de los muros que conforman el inmueble, tienen un espesor que va de los $45 \mathrm{~cm}$ a $\operatorname{los} 65 \mathrm{~cm}$.

Figura 1. Plantas arquitectónicas baja y alta, del inmuebles caso de estudio
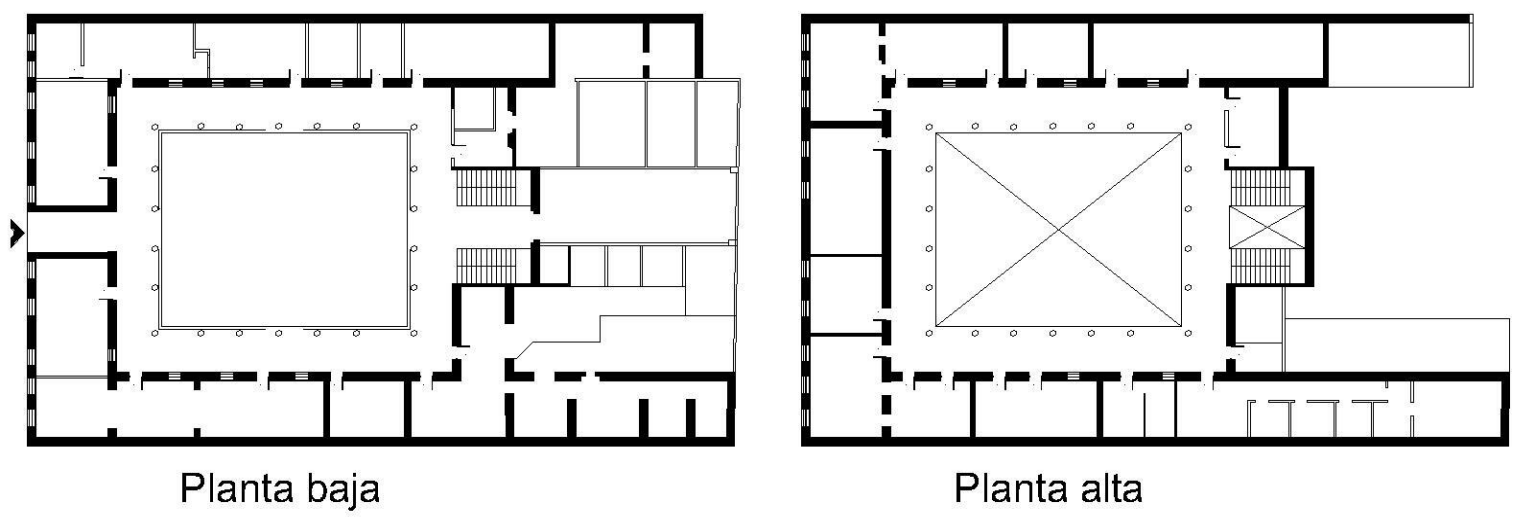

Fuente: Elaborado por el autor 
El sistema constructivo de la losa de entrepiso actualmente es una mezcla entre viguería con tapa de ladrillo y una losa de concreto aligerada, con espesor de 15 a $20 \mathrm{~cm}$. La losa de azotea presenta un sistema constructivo de vigueta $\mathrm{y}$ bovedilla. Referente a la dimensiones de los espacios interiores del inmueble, la planta baja cuenta con una altura de piso a entrepiso de 5.45 m., y la segunda planta de piso a techo tiene una altura de $4.65 \mathrm{~m}$. No obstante, con la instalación de entrepisos de falso plafón, para la colocación de sistemas de enfriamiento y de instalaciones sanitarias e hidráulicas, se cuenta con una altura de piso a entrepiso de $2.68 \mathrm{~m}$. en planta baja, y $2.69 \mathrm{~m}$. de altura en planta alta.

Para realizar el análisis higrotérmico, se comenzó por realizar una macro y una micro localización. Por medio de la macro localización se recopiló información referente al clima, vientos dominantes y asoleamiento de la zona de estudio, empleando la herramienta The weather tool, donde se obtuvo que los vientos dominantes tienen una dirección predominante hacia el oeste y suroeste. Con respecto al asoleamiento, el sol mostró un recorrido con inclinación hacia el sur. Para lo obtención de la información sobre el tipo de clima (Figura 2), se recurrió tanto a la consulta del Atlas Nacional de México (1990-1992), el cual está basado en el sistema de clasificación climática de Köppen modificada por Enriqueta García. Con la herramienta Meteonorm fue posible obtener información para definir las temperaturas medias mensuales durante el año en Culiacán (Figura 02). Así mismo, se determinaron los tres meses correspondientes a la temporada más caliente y tres meses de la temporada más fría (Figura 2), lo cual fue útil para definir los meses en los cuales se realizarían las mediciones de temperatura y humedad relativa, necesarias para el análisis higrotérmico.

La micro localización permitió analizar las condiciones de asoleamiento y vientos dominantes, utilizando la herramienta The weather tool, con respecto a la orientación del inmueble, la cual es hacia el sur. Para el estudio del asoleamiento, se realizó un análisis de la posición del sol durante los solsticios y equinoccios, tomando la posición solar a las 12:00 p.m., por medio de lo que se determinó que el recorrido solar durante todo el año presenta una inclinación predominante hacia el sur. El análisis de los vientos dominantes se realizó para obtener la variación de dirección de los vientos dominantes durante las cuatro estaciones del año, y posteriormente compararlo con la orientación del inmueble. Este análisis mostró una predominancia de los vientos provenientes desde el sur, lo cual, comparada con la orientación del inmueble, resultaría favorable para generar ventilación natural.

Para la recopilación de los datos de temperatura del aire y humedad relativa del inmueble, se recurrió a la monitorización por medio de medidores in situ, puesto que de acuerdo a la revisión del estado del arte, este método resulta adecuado para obtener datos confiables. La monitorización se llevó a cabo en dos periodos distintos, con un mes de duración cada uno, las veinticuatro horas del día. Estos periodos fueron elegidos de acuerdo a las temperaturas medias mensuales que se presentan en Culiacán, con lo que se seleccionaron dos meses, uno en la temporada cálida y otro en la temporada fría, siendo los meses de agosto y febrero respectivamente. Así mismo, el periodo de análisis de las monitorizaciones dependerá tanto del clima, como de la disponibilidad de tiempo y la facilidad para colocar los equipos en los

Figura 2. Temperaturas medias mensuales durante el año en Culiacán

\begin{tabular}{|c|c|c|c|c|c|c|c|c|c|c|c|c|}
\hline Mes & E & F & M & A & M & J & J & A & S & O & N & D \\
\hline $\begin{array}{c}\text { Temperatura } \\
\text { Media }\left({ }^{\circ} \mathrm{C}\right)\end{array}$ & $\mathbf{1 9 . 2}$ & $\mathbf{2 0 . 3}$ & 22.2 & 24.9 & 27.4 & 29.8 & $\mathbf{2 8 . 6}$ & $\mathbf{2 8 . 1}$ & 27.9 & 26.3 & 22.5 & $\mathbf{1 9 . 9}$ \\
\hline HR \% & 58 & 58 & 62 & 66 & 68 & 65 & 70 & 73 & 70 & 63 & 63 & 61 \\
\hline
\end{tabular}

Fuente: Elaborado por el autor 
espacios. En este caso, se propone un periodo de monitorizaciones considerado long-term.

Los instrumentos empleados fueron herramientas de medición llamados data loggers (HOBOS y HOBOS Pro v2), utilizando tres medidores interiores y un medidor exterior. Además, fueron utilizados datos de los mismos periodos monitoreados, de una estación meteorológica local para realizar comparación de datos. Para la selección de los espacios interiores donde se colocarían los medidores, se buscaron aquellos que se encontraran en funcionamiento, que no contaran con ningún tipo de acondicionamiento artificial, y que tuvieran distintas orientaciones, seleccionado así tres espacios interiores y uno exterior (Figuras 3 y 4 p. 38).

Los medidores interiores fueron colocados entre $1.70 \mathrm{~m}$. y $1.90 \mathrm{~m}$. de altura para interferir lo menos posible con las actividades diarias realizadas en el inmueble (Becerra-Santacruz y Lawrence, 2016), y se buscó que su colocación no estuviera cerca de corrientes de aire, en un sitio donde no recibiera rayos solares directos, ni cerca de aparatos electrónicos que generen calor, puesto que esto podría afectar y alterar las mediciones. Así mismo, se colocó un data logger en la azotea durante el mes de agosto, mientras que en el mes de febrero fue colocado en el patio central, para la obtención de datos el microclima exterior del inmueble.

Para realizar la validación de los data loggers y asegurar la confiabilidad de los datos, se siguió el método expuesto por Becerra (2016), colocando los medidores en una habitación cerrada, sobre una superficie plana a una altura de aproximadamente 1 metro, con una separación de 20 centímetros entre cada uno, tomando mediciones durante un día cada hora, los datos obtenidos nunca se tuvieron una diferencia, de un Data logger a otro, de más de 0.5 grados centígrados, lo cual se encuentra dentro del parámetro establecido. Con esto fue posible comprobar que las herramientas se encontraban con un funcionamiento adecuado para realizar los monitoreos.

Figura 3. Localización de los data loggers en el mes de agosto

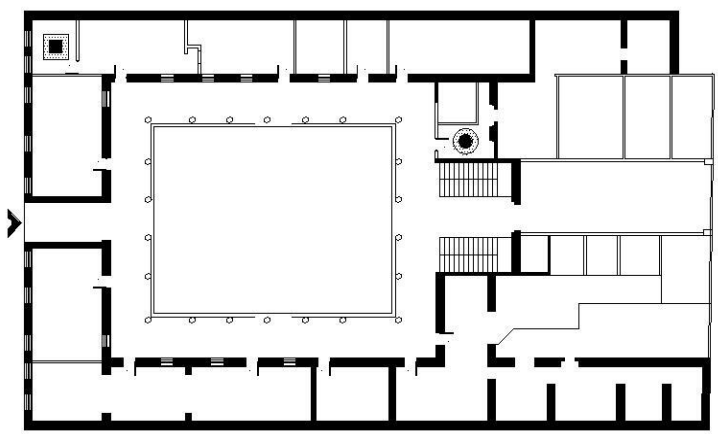

Planta baja

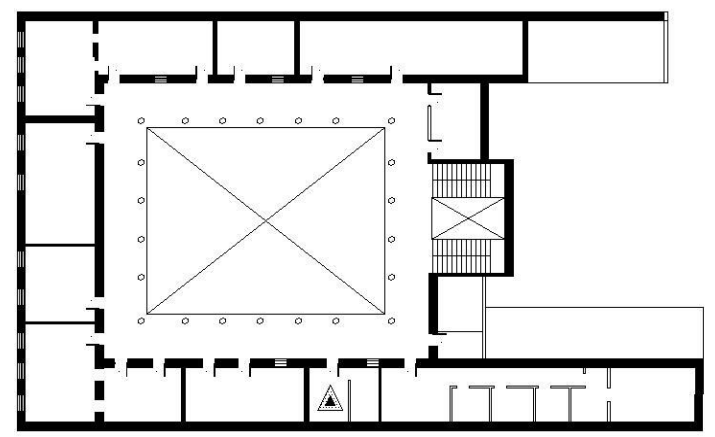

Planta alta

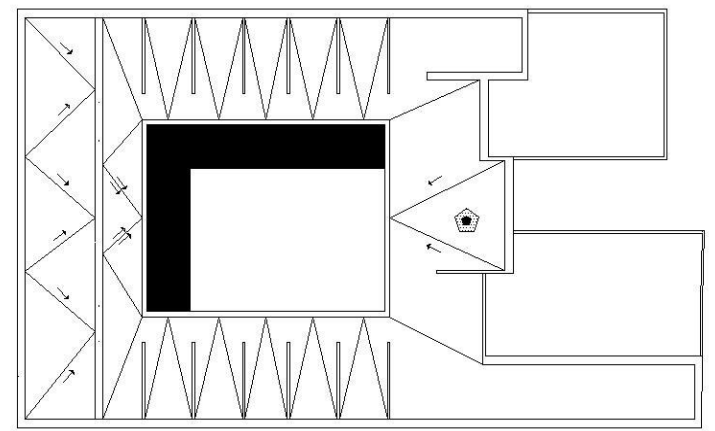

Planta de azotea

Hobo AH-01: Bodega biblioteca

Hobo AH-02: Fondos históricos

Hobo AH-03: Bodega planta alta

Hobo AH-04: Azotea

Fuente: Elaborado por el autor 
Figura 4. Localización de los data loggers en el mes de febrero

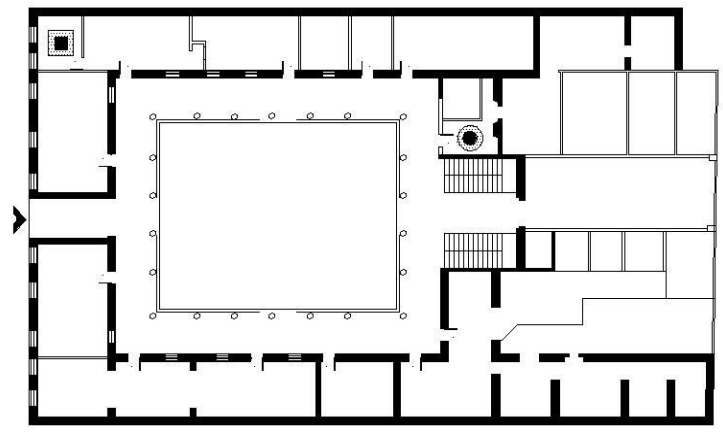

Planta baja

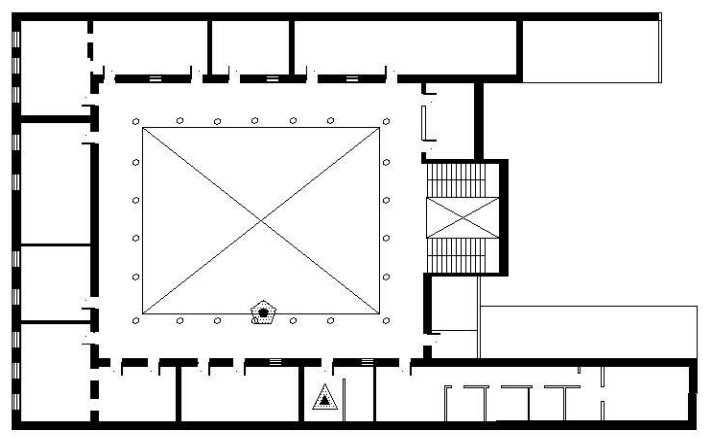

Planta alta

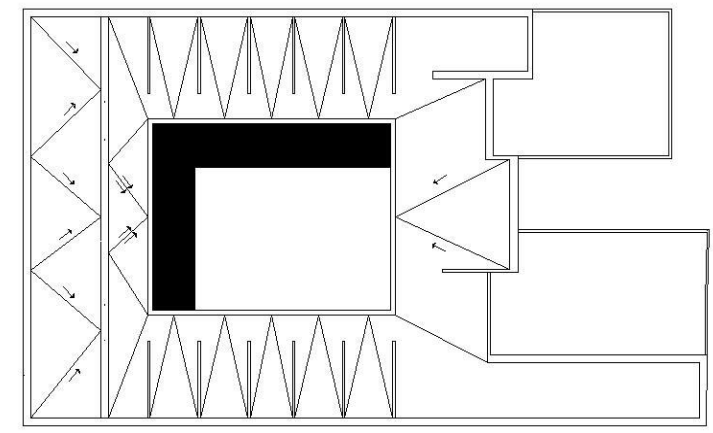

Planta de azotea

Hobo AH-01: Bodega biblioteca

Hobo AH-02: Fondos históricos

Hobo AH-03: Bodega planta alta

Hobo AH-04: Crujía este

Fuente: Elaborado por el autor

\section{RESULTADOS Y DISCUSIÓN}

Una vez recopilados los datos, estos fueron tratados para obtener promedios por hora, tanto de la temperatura del aire, como de la humedad relativa. Para la obtención del índice de confort, se siguió el modelo de Mauricio Roríz (2003), por medio del cual es posible obtener el rango de confort adaptativo por hora. Se seleccionó este modelo como el indicado por que brinda un rango de confort variable durante el día, a diferencia de otros modelos donde se establece un rango de confort estable, lo cual resulta importante puesto que en cualquier ambiente interior o exterior, se presenta una variabilidad temporal, es decir, no se tiene una temperatura fija durante todo el día.

Se presenta una franja de tolerancia de $+-2.5^{\circ}$ $\mathrm{C}$, y suponiendo que la variación de la línea de confort corresponda al $40 \%$ de la amplitud de la variación externa, se determina una zona de confort variable de una amplitud de $5^{\circ} \mathrm{C}$, con un límite superior y uno inferior. Con esto, se puedo determinar los periodos de superávit y los periodos en confort en las temperaturas obtenidas en los diferentes espacios, durante las dos temporadas (Figuras 5 y 6 p. 39).

Para el análisis de la humedad relativa, el parámetro de confort seguido es el expuesto en la versión vigente del RITE, donde se especifica que para condiciones interiores, en verano la humedad relativa deberá encontrarse entre un $45-60 \%$, y en invierno deberá ser de 40-50\%. Los resultados del análisis de humedad relativa se muestran sintetizados en dos gráficas correspondientes al mes de agosto y febrero (Figura 7 y 8 p. 40), se muestran los promedios por hora durante cada mes, y se comparan con la franja de humedad relativa confortable. 
Figura 5. Gráfica de análisis de temperaturas con respeto a la zona de confort adaptativo durante agosto

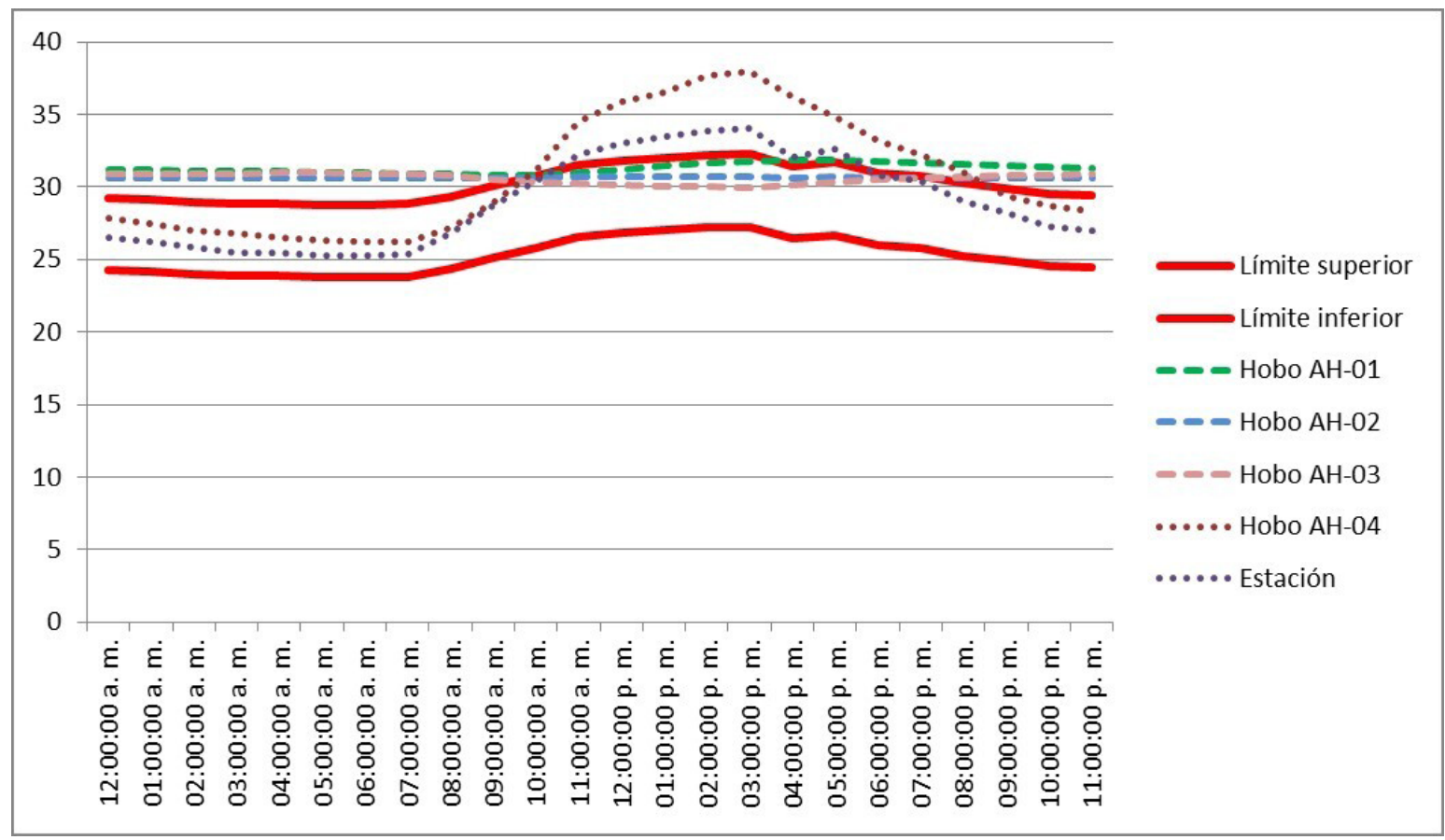

Figura 6. Gráfica de análisis de temperaturas con respeto a la zona de confort adaptativo durante febrero

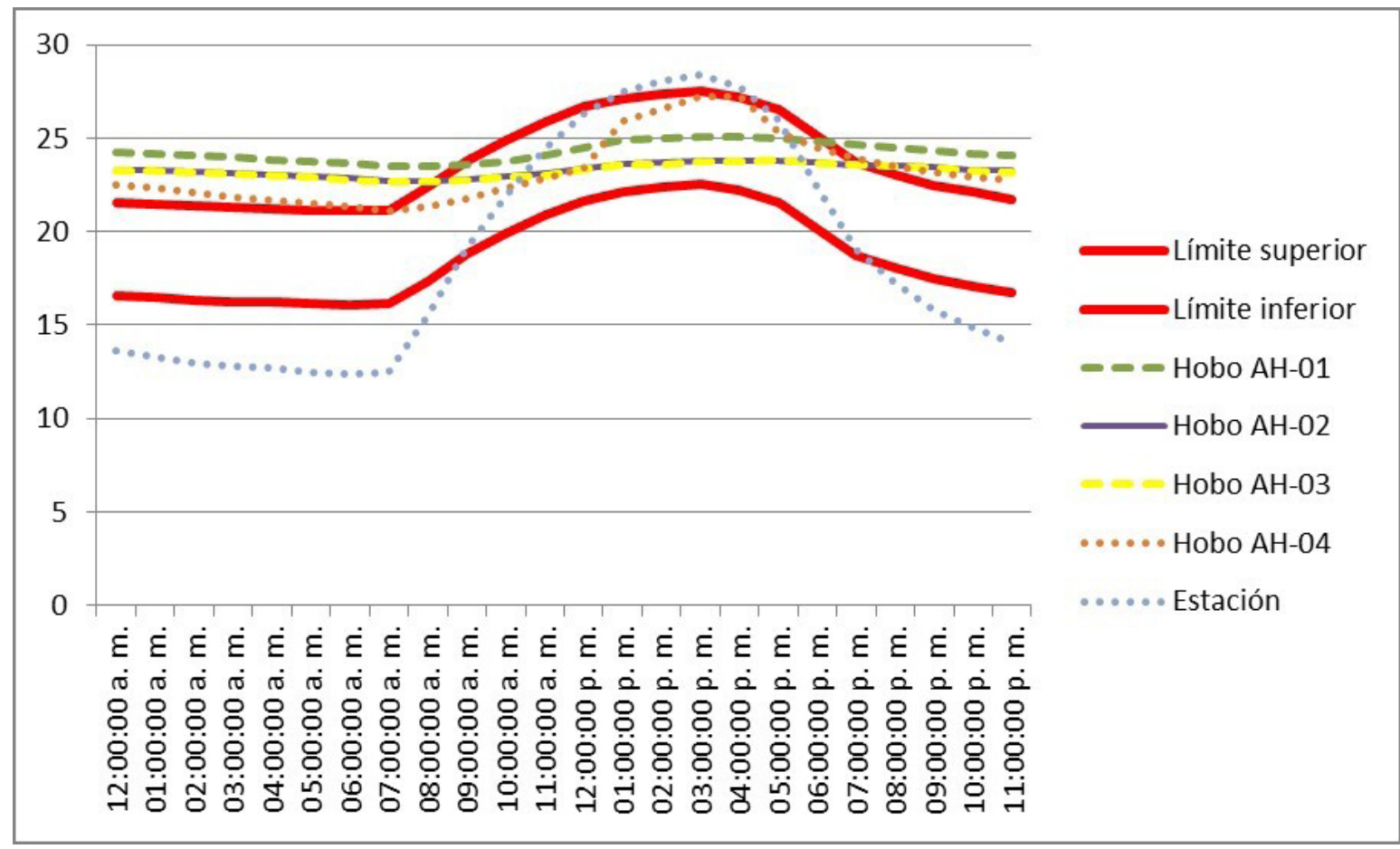

Fuente: Elaboradas por el autor 
Figura 7. Análisis de la humedad relativa en agosto, de acuerdo a los porcentajes de humedad relativa

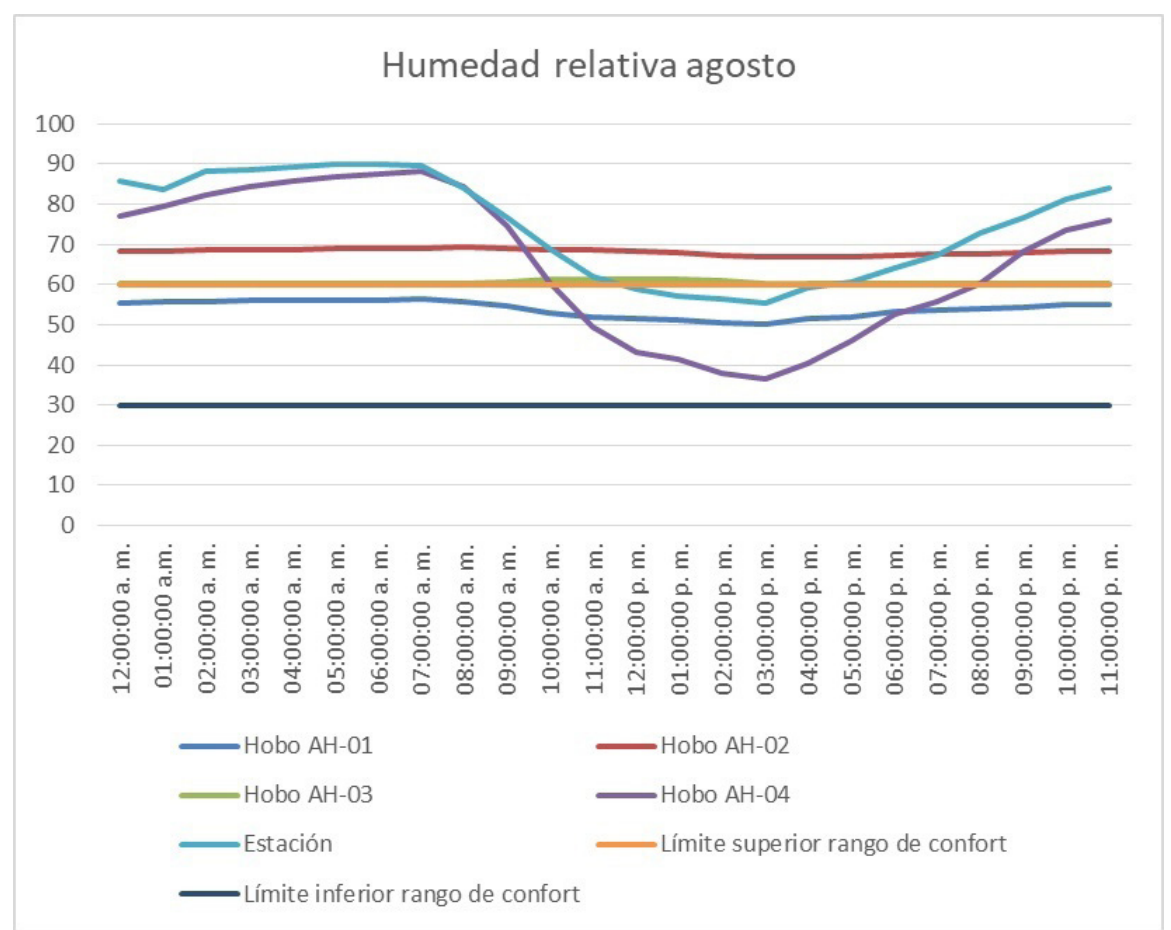

Figura 8. Análisis de la humedad relativa en febrero, de acuerdo a los porcentajes de humedad relativa

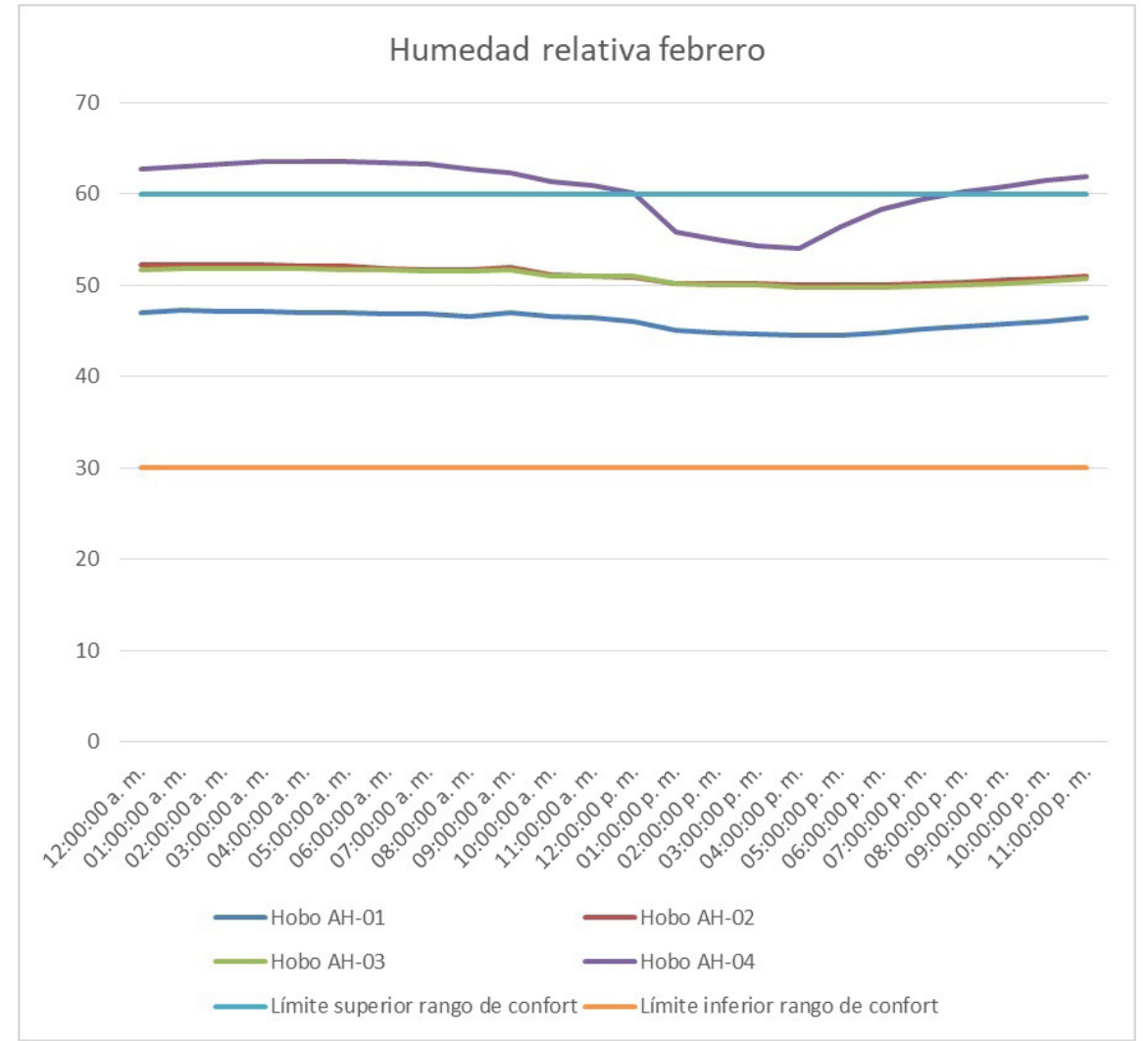

Fuente: Elaboradas por el autor 
Se obtiene que, tanto la temperatura del aire como la humedad relativa en el interior del inmueble (Hobo AH-01, Hobo AH-02 y Hobo AH-03) presentan un comportamiento constante, donde la temperatura del aire en su mayoría muestra variaciones de $1{ }^{\circ} \mathrm{C}$. El espacio interior donde se presenta mayor variación de temperatura y datos más elevados durante el día, es el ubicado con orientación sur (Hobo AH-01), mismo que por su orientación registra mayor incidencia de rayos solares. Sin embargo, es el espacio que presenta mediciones de humedad relativa confortable durante todo el día. En los espacios donde se colocaron los medidores Hobo AH-02 y Hobo AH-03, se recopilaron datos que muestran que el porcentaje de humedad relativa se encuentra en el límite superior, y en algunos casos por fuera de este.

Con respecto a la información recopilada con el Hobo AH-04, referente al microclima exterior del inmueble, este muestra que el comportamiento de la temperatura del aire presenta un comportamiento similar al de la estación meteorológica, pero con una curva de variación menos pronunciada. Mientras que la humedad relativa durante el mes de agosto, si bien es un comportamiento similar al de la estación meteorológica, los datos presentan una curva más pronunciada que la estación meteorológica. La humedad relativa en el interior durante el mes de agosto se encuentra en un rango confortable de 9:00 a.m. a 8:00 p.m. aproximadamente. Durante el mes de febrero no fue posible obtener datos de humedad relativa de la estación meteorológica, no obstante, de acuerdo a los comportamientos anteriores, la humedad relativa de la estación meteorológica podría presentar un comportamiento similar a la del Hobo AH-04, la cual se encuentra fuera del rango de confort.

Es importante señalar que el horario operacional del inmueble se extiende de 9:00 a.m. a 8:00 p.m. aproximadamente, realizándose actividades en su mayoría sedentarias. Esta información resulta relevante puesto que el comportamiento de la temperatura del aire en Hobo AH-01, Hobo AH-02 y Hobo AH-03, de acuerdo al rango de confort adaptativo, se encontraría dentro de este rango durante la mayor parte del horario operacional del inmueble.
Diagnóstico del comportamiento higrotérmico del inmueble

Referente a la variable temperatura del aire, se encontró que, de acuerdo al rango de confort adaptativo, existe un sobrecalentamiento en el interior, no obstante, es importante considerar el horario operacional del inmueble, puesto que la mayor parte del superávit se presenta fuera de este horario. Si bien se presenta necesidad de enfriamiento tanto en la temporada cálida como en la temporada fría, las horas que se requiere enfriar de acuerdo al horario de operación del inmueble son muy pocas. Tomando esto en consideración, podría disminuirse el uso de los sistemas de enfriamiento durante el día, lo cual contribuiría a minimizar el gasto energético del inmueble.

Por otro lado, los espacios interiores que cuentan con orientación sur muestran un aumento de la temperatura del aire en el interior del inmueble, atribuible a la incidencia de los rayos solares por la falta de estrategias para minimizar las ganancias solares por los vanos. Se requiere proteger contra la incidencia de los rayos solares para disminuir la temperatura.

Referente a la variable de humedad relativa, en el mes de agosto correspondiente a la temporada cálida, se presenta necesidad de deshumidificar en su mayoría, con excepción de los espacios con orientación sur, donde se cuenta con buena ventilación y mayor incidencia de los rayos solares.

De acuerdo al diagnóstico obtenido por medio del análisis higrotérmico, y de acuerdo a King (1994) y Fuentes (s.f.), se proponen posibles estrategias a implementar en el inmueble que minimicen el flujo conductivo de calor, minimicen las ganancias solares y favorezcan el flujo del viento, considerando que el problema principal que se tiene es el sobrecalentamiento, la falta de ventilación natural, y humedad. Obteniendo la siguiente lista:

- Implementación de aleros y parteluces

en fachadas

- Uso de tragaluces con control solar en verano

- Sistema de aislamiento térmico con

poliuretano expandido en muros

- Instalación de aislamiento en cubierta

(Barro Kappa)

Ventilación cruzada

Colocación de deshumidificadores 
- Colocación de plantas deshumidificadoras

- Paneles solares de celdas fotovoltaicas

No obstante, al tratarse de un inmueble histórico con valores patrimoniales que además se encuentra protegido por una ley federal, resulta necesario evaluar estas estrategias respecto al respecto de los valores patrimoniales. Se siguió el método utilizado por el estándar Europeo prEN 16883:2015, el cual considera el respeto al patrimonio como una categoría de evaluación, tomando en cuenta tres apartados: el impacto de los materiales, el impacto visual, el impacto espacial de las estrategias.

Así mismo, se le realiza una modificación, agregando un apartado para determinar el cumplimiento de la Ley federal sobre monumentos y zonas arqueológicos, artísticos e históricos. Son retomados de lo establecido en el artículo 45 del Reglamento de la Ley federal sobre monumentos y zonas arqueológicos, artísticos e históricos: No afecta el estado de conservación de los elementos arquitectónicos, y El funcionamiento de las instalaciones y servicios no altera ni deforma los valores del monumento. Es importante señalar que, este método se sustenta en un criterio de evaluación subjetivo, por lo cual se recomienda someter aquellas estrategias que suponen una intervención mayor en el inmueble, a evaluaciones adicionales que corroboren su idoneidad.

En este caso, las estrategias de sistema de aislamiento térmico con poliuretano expandido en muros, y la instalación de aislamiento en cubierta, se evaluaron por medio del uso de una herramienta computacional de simulación llamada EnerHabitat, la cual realiza una simulación numérica de transferencia de calor dependiente del tiempo, y permite comparar el desempeño térmico de sistemas constructivos de techos y muros de la envolvente del inmueble.

Referente al sistema de aislamiento térmico con poliuretano expandido en muros, se encuentra que, al contar con muros de grandes espesores en el sistema constructivo actual, al agregarle un sistema aislante de $0.15 \mathrm{~m}$. a $0.20 \mathrm{~m}$., no se podrá observar un cambio en el desempeño térmico del sistema constructivo. Podría necesitarse un sistema aislante con mayores espesores, sin embargo, esto no es recomendable ya que modificaría las dimensiones de los espacios, alterando la percepción visual y el espacio de trabajo (Borbolla, 2017). Respecto al aislante térmico en azotea, se propone el material denominado Barro Kappa, el cual está elaborado de barro natural, y es sobrepuesto en la azotea creando una capa térmica que aísla el calor.

Con la simulación, el sistema constructivo propuesto con el aislante térmico presenta un comportamiento lineal, encontrándose durante todo el día dentro de la zona de confort sin cambios bruscos de temperatura, al contrario del comportamiento que presenta el sistema constructivo actual, por lo cual, el aplicar este material aislante en la losa de azotea podría ser beneficioso en el comportamiento térmico del inmueble, puesto que se puede observar una disminución de temperatura. Por otro lado, al ser un material de fácil instalación y con un criterio de reversibilidad, se recomienda el uso de este (Ibídem).

En este sentido, se concluye que pueden ser utilizados métodos pasivos para evitar la ganancia de calor y favorecer las pérdidas de este (Fuentes, s.f.). Algunas estrategias pasivas recomendables serían hacer uso de la ventilación cruzada con ventanas operables que den a patios interiores, implementación de aleros y parteluces en fachadas, uso de vegetación en patios interiores para sombreado y deshumidificación, uso de tragaluces con control solar en verano, e instalación de aislamiento en azotea (Borbolla, 2017).

\section{CONCLUSIONES}

La rehabilitación energética de inmuebles históricos es el equilibrio entre eficiencia energética, confort de los usuarios y respeto a los valores patrimoniales. Es importante continuar generando datos e información respecto al comportamiento higrotérmico de los inmuebles históricos, como primer paso para evidenciar la necesidad de considerar esta clase de intervenciones.

El caso de estudio muestra que se podrían alcanzar temperaturas confortables de acuerdo al tipo de actividades que se realizan en el edificio, por medio de estrategias pasivas y poco invasivas. No obstante, se está recurriendo a la instalación de sistemas de enfriamiento, que representan parte importante del consumo energético del inmueble, y que contribuyen al deterioro de este. Así mismo, se debe señalar si se quiere mejorar las condiciones higrotérmicas de este tipo de inmuebles, mientras se respeten los valores patrimoniales y se disminuya el consumo energético, es importante combinar los análisis higrotérmicos con otro tipo de análisis que ayuden a encontrar las estrategias más adecuadas para la conservación del inmueble. 
El análisis del comportamiento higrotérmico de inmuebles históricos como una etapa en el proceso de rehabilitación energética resulta una herramienta clave para la toma de decisiones que contribuyan a la mejora de la eficiencia energética, permitiendo que este tipo de inmuebles formen parte de las medidas de adaptación y mitigación para combatir el cambio climático en las ciudades, y evitando que se realicen intervenciones que se encuentren en conflicto con la conservación de los inmuebles históricos, logrando llegar a un equilibrio sumamente necesario. En este sentido, si bien se necesita generar más información en esta área de estudio, debido a las características particulares de cada inmueble, se presenta como un nicho de estudio útil en el campo de la conservación patrimonial que traería beneficios importantes y necesarios a nivel ecológico y arquitectónico. C

\section{REFERENCIAS BIBLIOGRÁFICAS}

Ballart, Josep. (2002). El patrimonio histórico y arqueológico: valor y uso, Barcelona: Ariel.

Becerra-Santacruz, Habid. (2016). Low income housing in México: An evaluation of thermal confort and performance of industralised housing construction system in warm temperate climate (Tesis doctoral). Universidad de Sheffield, Sheffield.

Becerra-Santacruz, Habid., y Lawrence, Ranald. (2016). "Evaluation of the thermal performance of an industrialised housing construction system in a warmtemperate climate: Morelia, Mexico", Building and Environment, 107, pp. 135153. Doi:10.1016/j.buildenv.2016.07.029

Borbolla Gaxiola, Catalina. (2017). Propuesta de metodología para rehabilitación energética de inmuebles patrimoniales. Caso de estudio: Archivo Histórico en Culiacán, Sinaloa (Tesis de maestría). Universidad Michoacana de San Nicolás de Hidalgo, Morelia, Michoacán.

Broström, T. y Leijonhufvud, G. (2010) "The Indoor Climate in Skokloster Castle. D. Del Curto" (Ed.), Historical buildings as museums: Systems for climate control and heritage preservation. Florencia, Italia: Department of Conservation. pp. 84-93.

Cámara de Diputados del H. Congreso de la Unión. (2015). Ley de transición energética. Recuperado de http://www.diputados.gob. $\mathrm{mx} /$ LeyesBiblio/pdf/LTE.pdf
Claesson, M. y Brostöm, T. (2016). Eight years of energy efficiency in historic buildings, Segunda Conferencia Internacional sobre Eficiencia Energética y Confort en Edificios Históricos. Conferencia llevada a cabo en Bruselas, Bélgica.

Curtis, R. (2016). Energy efficiency in traditional and historic buildings: keeping it simple, Segunda Conferencia Internacional sobre Eficiencia Energética y Confort en Edificios Históricos. Conferencia llevada a cabo en Bruselas, Bélgica.

De bouw, M., Dekeyser, L., Dubois, S. y Vanhellemont, Y. (2016) Second International Conference on Energy Efficiency and comfort of historic buildings, Bruselas, Flanders Heritage Agency.

Dubois, S. de Bouw, M., Vanhellemont, Y., Stiernon, D. y Trachte, S. (2018) Combining multi-view photogrammetry and wireless sensor networks when modelling the hygrothermal behaviour of heritage buildings, Tercera Conferencia Internacional de Eficiencia Energética en Edificaciones Históricas. Conferencia llevada a cabo en Visby, Suecia. Recuperado de: https://www. iea-shc.org/Data/Sites/1/publications/ Energy-efficiency-in-historic-buildings preliminary-conference-report.pdf

Egusquiza, Aitziber, Prieto, Iñaki. Izkara, José Luis y Bejar, Rubén. (2018) "Multi-scale urban data models for early-stage suitability assessment of energy conservation measures in historic urban áreas", Energy \& Buildings, 164, pp. 87-98.

Epelde Merino, Marta. (2015) Soluciones de aislamiento térmico para edificios protegidos de especial interés arquitectónico o histórico. Ejemplo de actuación, Sexta Conferencia Europea sobre eficiencia energética y sustentabilidad en arquitectura y planeación. Conferencia llevada a cabo en Donostia, San Sebastián.

Espinoza, José, Quesada, Felipe, Calle, Andrea y Ortiz, Jessica. (2019) "Estándares de sustentabilidad para viviendas patrimoniales del Centro Histórico de Cuenca-Ecuador", Estoa, 8 (15), pp. 65-78

Fuentes Freixanet, Víctor Armando (s.f.). Arquitectura Bioclimática. , Universidad Autónoma Metropolitana- Azcapotzalco 
González Licón, Héctor Javier (2006). Vivienda tradicional de la región Purhépecha. Adecuación al medio ambiente, espacios $y$ configuración formal, (Tesis de Doctorado). Programa Interinstitucional de Doctorado en Arquitectura, Facultad de Arquitectura, Colima.

Granados Menéndez, Helena. (2014). Rehabilitación Energética de Edificios. Madrid: Tornapunta

Gregório, Vera y Seixas, Júlia. (2017). "Energy savings potential in urban rehabilitation: A spatial-basedmethodology applied to historic centres", Energy and Buildings, 157, 11-23. Doi: http://dx.doi. org/10.1016/j.enbuild.2017.06.024

Guerra-Santin, Olivia y Tweed, Aidan Christopher. (2015) "In-use monitoring of buildings: An overview and classification of evaluation methods", Energy \& Buildings, (86), pp. 176-189. Doi: https://doi.org/10.1016/j. enbuild.2014.10.005

ICOM (2008) Terminología para definir la conservación del patrimonio cultural tangible, Nueva Delhi.

Jara, Paola. (2015) "Confort térmico, su importancia para el diseño arquitectónico y la calidad ambiental del espacio interior", Revista Arquitectura y Cultura, (7), 106121. Recuperado de: http://www.revistas. usach.cl/ojs/index.php/amasc/article/ view/2529

King Binelli, Delia. (1994) Acondicionamiento Bioclimático, Ciudad de México: Universidad Autónoma Metropolitana.

Krus, M., Kilian, R. y Pfundstein, B. (2016) Comparison of different systems for internal Wall insulation with reversible application for historic buildings, Segunda Conferencia Internacional sobre Eficiencia Energética y Confort en Edificios Históricos. Conferencia llevada a cabo en Bruselas, Bélgica.

López-Zambrano, M. J., Canivell, Jacinto y Calama-González, Carmen María (2019). "Sistema de evaluación de soluciones de rehabilitación energética para edificios bien de interés cultural (SESREBIC). Su aplicación a monasterios BIC", Informes de la construcción, 71 (555), pp. 01-12

Liudmila, Cazacova y Balkiz, Yapicioglu (2019). "Bridging Sustainable Construction Technologies and Heritage: Novel
Approach to the Conservation of the Built Environment". En V. Tam y K. Le (Ed), Sustainable Construction Technologies. Life-Cycle Assessment. Australia: Butterworth-Heinemann, pp. 395-442.

Lucchi, E., Garegnani, G., Maturi, L. y Moser, D. (2014). Architectural integration of photovoltaic system in historic districts. The case of study of Santiago de Copostela, Congreso Internacional de Eficiencia Energética y Edificación Histórica. Congreso llevado a cabo en Madrid, España.

Muñoz, C., León, A. y Navarro, J. (2014). La monitorización y simulación higrotérmica como herramienta para la mejora del confort, preservación y ahorro energético de espacios patrimoniales. El caso de la iglesia de San Francisco de Asís, Morón de la Frontera, Congreso Internacional de Eficiencia Energética y Edificación Histórica. Congreso llevado a cabo en Madrid, España.

Muranim, Ulisses y Ghisi, Enedir (2016). "Environmental feasibility of heritage buildings rehabilitation", Renewable and Sustainable Energy Reviews, 58, pp. 235-249. Doi: http://dx.doi.org/10.1016/j. rser.2015.12.334

Riegl, Alois (1987). El culto moderno a los monumentos, Madrid: Visor.

Roríz, Mauricio. (2003). Flutuacoes horárias dos limites de conforto térmico: Una hipótese de modelo adaptativo, Memórias de ECAC COTEDI.

Santos, A., Valenca, P. y Sequeira, J. (2014). Energy efficiency and urban renewal of a UNESCO- listed historical center: The case of Porto, Congreso Internacional de Eficiencia Energética y Edificación Histórica. Congreso llevado a cabo en Madrid, España.

Sassine, Emilio. (2016). "A practical method for in-situ thermal characterization of walls", Case Studies in Thermal Engineering, 8, pp. 84-93. Doi: https://doi.org/10.1016/j. csite.2016.03.006

Serra Florensa, Rafael y Coch Roura, Helena (1995). Arquitectura y energía natural, Barcelona: UPC.

Troi, A. (2016). Comfort and energy efficiency in historic buildings - the 3ENCULT experience, 
Segunda Conferencia Internacional sobre Eficiencia Energética y Confort en Edificios Históricos. Conferencia llevada a cabo en Bruselas, Bélgica.

Wessberg, M., Leijonhufvud, G. y Brostöm, T. (2016). An evaluation of three different methods for energy efficient indoor climate control in Skokloster Castle, Segunda Conferencia Internacional sobre Eficiencia Energética y Confort en Edificios Históricos. Conferencia llevada a cabo en Bruselas, Bélgica.

World Energy Council (2004) Energy Efficiency: A worldwide review. Indicadors, Policies, Evaluation, Londres, World Energy Council and French Environment and Energy Management Agency.

Yung, Esther H. K., y Chan, Edwin H. W. (2012). "Implementation challenges to the adaptive reuse of heritage buildings: Towards the goals of sustainable, low carbon cities", Habitat International, 36(3), pp. 352-361. Doi: https://doi. org/10.1016/j.habitatint.2011.11.001 